\title{
NUMERICAL MODELLING OF COMPRESSIVE CHARACTERISTICS OF AUXETIC STRUCTURES
}

\author{
Michaela Neuhäuserová*, Petr Koudelka \\ Czech Technical University in Prague, Faculty of Transportation Sciences, Department of Mechanics and \\ Materials, Konviktská 20, 11000 Prague 1, Czech Republic \\ * corresponding author: neuhauserova@fd.cvut.cz
}

\begin{abstract}
The paper is focused on numerical analysis of mechanical behaviour of auxetic structures with re-entrant tetrakaidecahedral unit cell subjected to uni-axial quasi-static compression. The mechanical behaviour was evaluated inversely with respect to selected geometrical parameters of the unit cell and two different loading modes. Finite element method was used for the numerical analysis of the problem. A set of fully parametric tools has been developed, which enabled automated execution and evaluation of virtual experiments. From results of the simulations, Young's modulus, the characteristics of the Poisson's ratio function, and the deformation energy density were estimated. The relation between these characteristics and geometry of the unit cell, particularly the re-entrant angle and the relative density, was evaluated. Results of the numerical simulations for the unit cell and representative volume element of its three-dimensional periodic assembly are presented.
\end{abstract}

KEYworDs: auxetic structures, re-entrant tetrakaidecahedron, finite element method, uni-axial loading, quasi-static compression.

\section{INTRODUCTION}

\subsection{BACKGROUND}

Porous solids, particularly metal foams, are nowadays often utilized in various kinds of applications due to their advantageous mechanical properties. For example, their relatively low specific weight and thus high specific strength, make them suitable for applications requiring mass reduction and simultaneous high energy absorption [1]. However, for constructions subjected to extreme ways of loading such as high strain-rate impacts or blasts, compressive properties of conventional metal foams are insufficient [2]. Thus, alternative materials are sought and investigated. One of possible approaches is development of optimized auxetic structures 3 .

\subsection{Auxetic Structures}

Auxetic materials are materials with negative Poisson's ratio. When stretched laterally, they get thicker in transverse direction and vice versa. Due to this unconventional deformation behaviour, auxetics have unique mechanical properties including the ability to absorb relatively high amount of deformation energy [4. There exist only a few known natural auxetic materials, majority of them are produced artificially based on geometric models with deformation behaviour analytically described in closed form [5] . Modern production methods such as additive manufacturing allows precise production of structures with controlled geometry. Therefore, these methods are used for production of auxetic materials based on known geometric models [6].

\subsubsection{RE-ENTRANT TETRAKAIDECAHEDRON}

Auxetic structure with unit cell in shape of re-entrant tetrakaidecahedron was first introduced by Choi and Lakes in 1995 [7. In their study, they presented analytical model of mechanical behaviour of the unit cell as well as simple evaluation experiment for irregular assembly of the unit cells. Both the analytical and the experimental data provided in the study indicated auxetic character of the structure with reentrant tetrakaidecahedral unit cells.

The geometry of the auxetic unit cell is a modification of conventional tetrakaidecahedron with square faces protruded in the inner space of the cell. Schemes of both, conventional and re-entrant tetrakaidecahedrons can be seen in Figure 1
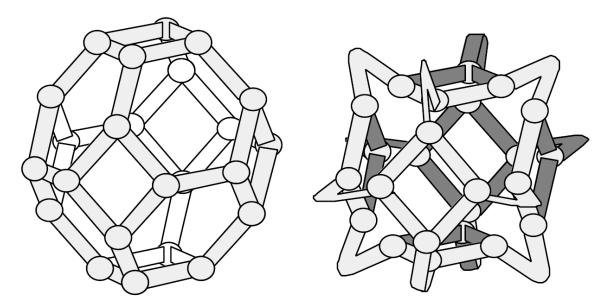

FigURE 1. Geometry of conventional tetrakaidecahedron (left) and re-entrant tetrakaidecahedron (right) 7

This geometry of the unit cell was for this study selected due to its spatial symmetry, which determines potential of auxetic structure created by threedimensional periodic assembly of this unit cell to be mechanically orthotropic 8 . Therefore, the structure can exhibit auxetic character in three orthogonal di- 
rections in contrast to other auxetics, which in most cases exhibit negative Poisson's ratio only in one or two orthogonal directions [5].

Mechanical behaviour of the re-entrat tetrakaidecahedral unit cell has been described by Choi and Lakes in [7. The model is based on geometrical aspects of deformations occurring in the structure if square faces of the cell is loaded. The equations 1 and 2 present the analytical model for elastic and for plastic deformations [7]. As can be seen from the equations, the model is based on the angles of the protruded ribs in the structure. The figure 2 illustrates the re-entrant angle $\varphi$. The angle $\theta$ represents the angle by which the rib $J K$ rotate around the point $K$ during larger deformations.

$$
\begin{aligned}
& \nu_{e l}=-\frac{\sin (\varphi-\pi / 4)}{\cos (\varphi-\pi / 4)} \\
& \nu_{p l}=-\frac{\cos (\varphi-\pi / 4-\theta)-\cos (\varphi-\pi / 4)}{\sin (\varphi-\pi / 4-\theta)-\sin (\varphi-\pi / 4)}
\end{aligned}
$$

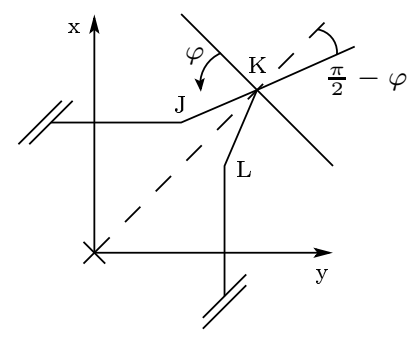

FiguRE 2. One quater of cross section of the re-etrant tetrakaidecahedral unit cell

\subsection{AIM}

The main aim of the study is to perform numerical analysis of microstructural characteristcs to inversely evaluate effective mechanical properties of auxetic structures with re-entrant tetrakaidecahedral unit cell. For this reason, simulated uni-axial quasi-static compression testing of the structures was performed using the Ansys finite element (FE) software.

\section{Numerical AnAlysis}

To perform the numerical analysis, a set of fully parametric tools has been developed in Ansys software and automated execution and evaluation of virtual experiments have been implemented.

\subsection{Geometric MOdels}

The numerical simulations were performed using two different geometric models of the structure. The unit cell of the re-entrant tetrakaidecahedron itself as well as a representative volume element (RVE) of the structure created by three-dimensional periodic assembly of the unit cells were analysed. The analysis of the unit cell itself was performed foremost for the comparison with the analytical model as well as for acquiring the knowledge of deformation processes on the elementary level of the structure. However, as can be seen in the Results section, the results obtained from analysis of RVE of the structure are rather predicative in terms of practical utilization of such a structure.

The dimensions of the unit cell itself were $2.3 \times$ $2.3 \times 2.3 \mathrm{~mm}$ since the dimension of a unit beam in the structure (one side of the rectangular face) is $1 \mathrm{~mm}$. The size of the RVE was estimated based on analysis of Young's modulus of structures created by increasing number of cells. For lower numbers, the value of Young's modulus significantly varied. The higher number of cells was, the Young's modulus of the structure rather converged to a specific value and lowest number of cells for which the value converged was 12 along each axis. Therefore, the RVE contained 12 unit cell along each axis yielding 1728 unit cells in total. The dimensions of the RVE were $27.6 \times 27.6 \times$ $27.6 \mathrm{~mm}$.

The geometric models were generated with variable parameters such as the size of the re-entrant angle and the relative density of the structure. The size of the re-entrant angle determines the rate of protrusion of rectangular faces into the inner space of the unit cell (the larger the angle is, the more the cell is enclosed). The relative density is given by the volumetric ratio of solid mass in the structure and in the models, it is varied by changing the thickness of beams creating the structure. In the Figure 3 can be seen how different values of these parameters affect the overall design of the unit cell.

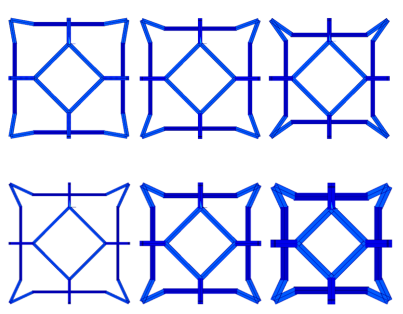

Figure 3. Top: The affect of increasing re-entrant angle on the design of the unit cell (from left to right). Bottom: The affect of increasing relative density on the design of the unit cell (from left to right)

\subsection{MATERIAL MODEL}

Construction material was simulated with isotropic, linear elastic material model. Its parameters, particularly Young's modulus and Poisson's ratio, were set according to values corresponding to powder stainless steel SS 316L-0407 by Renishaw. The values of the parameters are noted in Table 1 . This material was selected since it is commonly used for selective laser sintering (additive manufacturing method) and based on the results of the study, real samples of the structure are about to be produced using this specific material. 


\begin{tabular}{ll}
\hline Parameter & Value \\
\hline Young's modulus & $190 \mathrm{GPa}$ \\
Poisson's ratio & 0.27 \\
\hline
\end{tabular}

TABLE 1. Parameters of material model

\subsection{Finite Element Mesh and numerical BOUNDARY CONDITIONS}

In order to perform the numerical analysis, the geometric models were further transformed to the mesh of finite 3D beam elements with linear shape function and six degrees of freedom at each node.

Boundary conditions were specified at selected nodes of the models. All degrees of freedom were constrained at all nodes with maximal coordinate in one selected spatial direction forming one support in the virtual compressive experiment. In terms of loading, there were two different loading modes analysed. In the first mode, the loads were applied on the nodes in the vertices of square faces of the unit cell. In the second mode, the loads were applied on the nodes in the vertices of the triangular faces. Schemas with highlighted nodes with specified boundary conditions in both of the loading modes can be seen in Figure 4 . The loading procedure was displacement driven in both cases. In order to evaluate Young's modulus, the models were loaded in one load step up to $2 \%$ of deformation. The other parameters were estimated from loading in multiple load steps. The model of RVE was loaded in five load steps to the total deformation of $5 \%$ and the model of the unit cell was loaded in 30 load steps to the total deformation of $10 \%$.
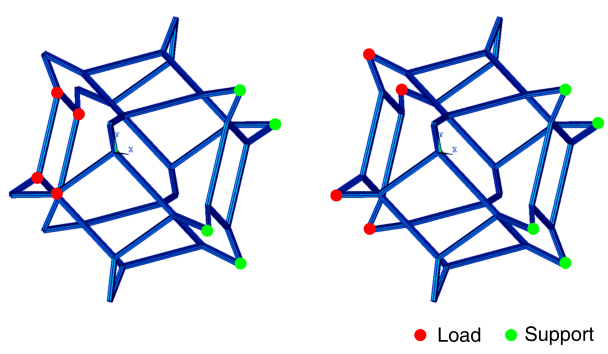

FIGURE 4. Boundary conditions in first loading mode (left) and in the second loading mode (right).

The first of the loading modes was analysed especially because the analytical model of the unit cell's deformation behaviour is based on this mode [7]. However, the second mode rather corresponds to the way in which the loads would be transferred into the structure during a real compression testing.

\section{RESUlts}

The results of the numerical simulations has been utilized for inverse estimation of selected material characteristics of the structures. Particularly, Young's modulus, or relative Young's modulus (the ratio of
Young's modulus of the structure and Young's modulus of the construction material), Poisson's ratio and the deformation energy density were evaluated with respect to specific geometrical parameters of the unit cell, such as the re-entrant angle and the relative density of the structure. Moreover, the mechanical behaviour of the structure was analysed with respect to two different loading modes.

\subsection{Relative YounG's modulus With RESPECT TO RELATIVE DENSITY}

The dependency of relative Young's modulus on relative density of the structure RVE with re-entrant angle $70^{\circ}$ was analysed in both loading modes. As can be seen at the resulting diagrams in Figure 5, the relative Young's modulus is in both cases proportional to the relative density of the structure and the curves have very similar trend for both loading modes.

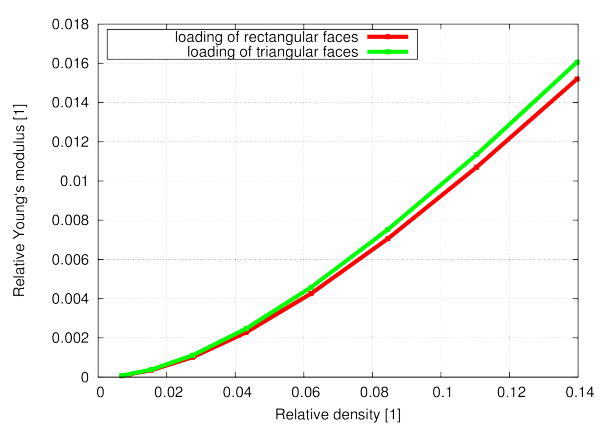

Figure 5. Relative Young's modulus of RVE of the structure plotted against its relative density

The acquired diagrams have similar trend to such diagrams for conventional porous solids [2]. However, the values are lower than values estimated for conventional foams with open cells e.g. Gibson-Ashby cells analysed in [9]. This trend corresponds to the assumption that auxetic structures have lower stiffness and thus also lower value of Young's modulus or relative Young's modulus than conventional porous solids [10.

\subsection{Poisson's RATio of the StruCture WITH RESPECT TO THE SIZE OF RE-ENTRANT ANGLE}

Then the dependency of Poisson's ratio on the size of re-entrant angle was analysed on the model of RVE with relative density equal to 0.02 . As can be seen on resulting diagrams in Figure 6, the values of Poisson's ratio are inversely proportional to the size of re-entrant angle in both loading modes. As can be also seen, for most of the geometries the value of Poisson's ratio was not negative within analysed deformation range. Nevertheless, the trend of the resulting diagram is in all cases descending and thus it is likely that negative values could be obtained at larger deformations.

It was not possible to perform the simulations of larger deformations due to the simplicity of the model which is used in this study. This model would not 
consider contacts of the parts during larger deformations. In order to simulate such tasks, the current model needs to be further modified.
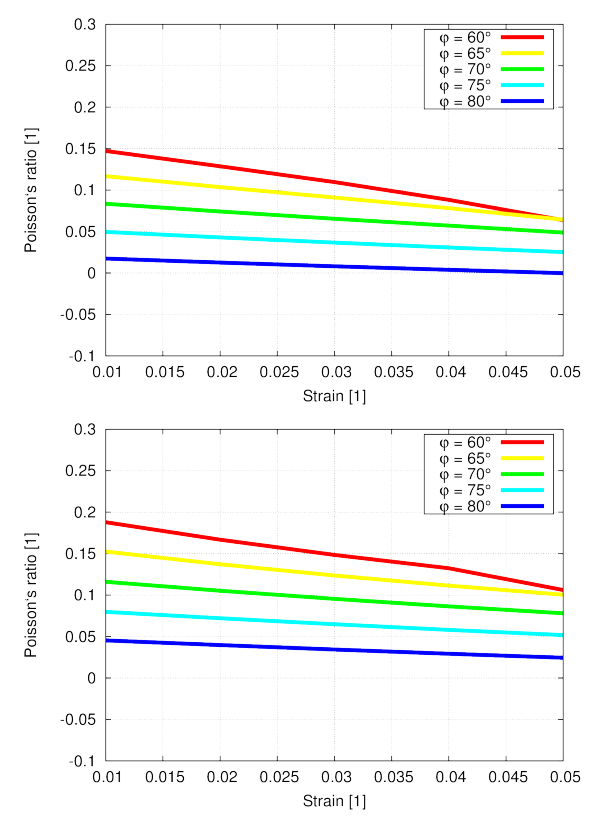

Figure 6. The Poisson's ratio function (strain dependent Poisson's ratio) of RVE with respect to the size of re-entrant angle in first loading mode (top) and second loading mode (bottom).

\subsection{Poisson's RAtio of the structure WITH RESPECT TO ITS RELATIVE DENSITY}

The Poisson's ratio function (strain dependent Poisson's ratio) for the RVE of the structure with reentrant angle of size $70^{\circ}$ was also analysed with respect to relative density of the structure. In Figure 7 are depicted resulting diagrams.

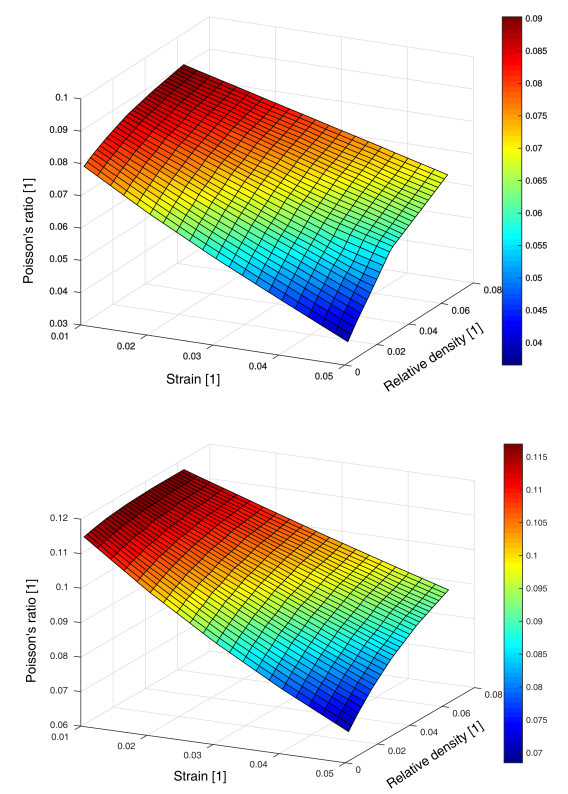

Figure 7. The Poisson's ratio function of RVE with respect to the relative density of the structure
It can be seen that the values of Poisson's ratio are directly proportional to relative density of the structure. This phenomenon occurs as a result of filling the total volume of the structure with the construction material. Thus, the range of deformations within the structure is limited.

\subsection{Deformation EnERgy DENSity}

The deformation energy density $\lambda$ is a mechanical characteristic representing the ability of material to absorb deformation energy and its value is given by the area under the stress-strain curve. The results obtained for RVE with relative density of 0.02 with respect to different re-entrant angles are denoted in the table below. The values of $\lambda_{1}$ corresponds to the deformation energy density from the first loading mode (loading of rectangular faces) and the $\lambda_{2}$ denotes the values for the second loading mode (loading of triangular faces).

\begin{tabular}{c|cc}
\hline Re-entrant angle $\varphi$ & $\lambda_{\mathbf{1}}\left[\mathbf{J} / \mathbf{m}^{\mathbf{3}}\right]$ & $\lambda_{\mathbf{2}}\left[\mathbf{J} / \mathbf{m}^{\mathbf{3}}\right]$ \\
\hline $60^{\circ}$ & 6732 & 118 \\
$65^{\circ}$ & 5973 & 91 \\
$70^{\circ}$ & 5245 & 73 \\
$75^{\circ}$ & 4660 & 56 \\
$80^{\circ}$ & 4119 & 49 \\
\hline
\end{tabular}

TABLE 2. Deformation energy density of RVE with respect to re-entrant angle size

The values of deformation energy density are inversely proportional to the size of re-entrant angle for both loading modes. Moreover, there is a significant difference between the values with respect to the loading mode. As has been observed from the models of the structure after deformation, the mechanical response of RVE differs for the loading modes. When the loads are applied on the vertices in rectangular faces, the structure "densifies" in the area where the loads are applied. In the other hand, when the vertices of triangular faces are loaded, the deformation is distributed gradually throughout the structure and displacements are spread layer by layer. Therefore, the energy accumulated in the structure is higher in the first case.

Similarly to conventional porous solids, auxetic structures have the potential to absorb relatively high amount of deformation energy especially due to significant stress plateau, which occurs after yield point [2]. However, the simulations in this study were performed only in a limited deformation range with only a linearelastic material model and thus this phenomenon can not be observed. Therefore, further investigation of larger deformation ranges on modified model of RVE is necessary in order to fully evaluate the ability of this structure to absorb deformation energy. 


\subsection{Evaluation OF THE ANALYTiCAL MODEL}

As has already been mentioned, the analytical model of auxetic unit cell in shape of re-entrant tetrakaidecahedron was first presented by Choi and Lakes in 1995 [7. Since then, the literature does not provide any further studies concerning such a unit cell or any structure based on this unit cell.

The comparison of the Poisson's ratio function estimated on the basis of the analytical model presented in [7] and the results obtained from the finite element analysis performed in this study can be seen in Figure 8. The analytical model is defined with load applied at the square face of the cell. Therefore, the results of numerical analysis of the first loading mode were used for the comparison.

It is obvious that in both cases, according to analytical model as well as finite element model, the Poisson's ratio of the structure is inversely proportional to the size of the re-entrant angle. Numerical results varies from the analytical significantly in the initial deformation zone up to approximately $4 \%$ of deformation. Nevertheless, for larger deformations the values of Poisson's ratio estimated numerically are similar to the analytical values. Given differences can be given by the nature of the analytical model, which is based only on the size of the re-entrant angle and does not assume any other parameters which can affect the mechanical behaviour of the structure.

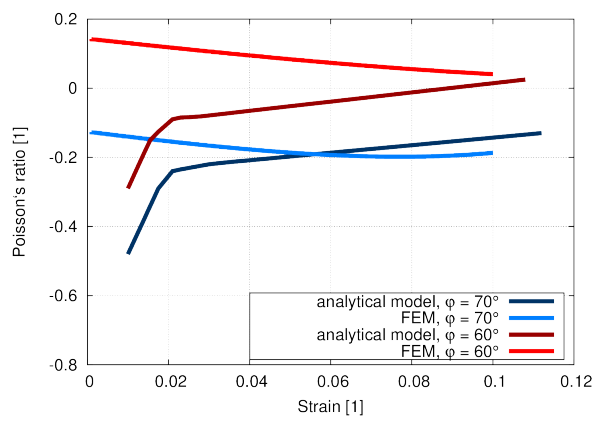

Figure 8. Comparison of Poisson's ratio functions obtained from analytical model [7] and from the finite element analysis performed in this study

\section{Conclusions}

This study was focused on numerical analysis of mechanical behaviour of auxetic structure with unit cell in shape of re-entrant tetrakaidecahedron. Simulated mechanical behaviour of the representative volume element of the structure as well as the unit cell itself were analysed. Selected mechanical properties, such as Young's modulus, Poisson's ratio and the deformation energy density were evaluated with respect to specific geometrical parameters of the unit cell, such as the re-entrant angle and the relative density of the structure. Moreover, the mechanical behaviour of the structure was analysed with respect to two different loading modes.
The results from the analysis of the unit cell were used for evaluation of analytical model of the cell. The values of Poisson's ratio obtained from the numerical simulations of deformations larger than $4 \%$ were similar to the analytical values and hereby the auxetic potential of such a geometry was numerically verified. However, further investigation of the structure is necessary in order to fully verify and maybe modify the analytical model due to the differences in the Poisson's ratio functions obtained from the analytical model and from the numerical simulations. E.g. verification experiments or more complex numerical analyses should be considered.

The results obtained from the analysis of the RVE were used to evaluate effective properties of periodical assembly of unit cells. The knowledge of the relation between the mechanical properties of the structure and its geometric parametes can be further utilized in development of optimization procedures [11. In such a process, optimal geometry of the structure corresponding to required mechanical properties can be proposed. Moreover, the unit cell geometry can be further modified in order to obtain deformation behaviour corresponding to loading of square faces across the whole periodic assembly of the cells. This can be achieved e.g. by adding stems between the square faces of the neighbouring unit cells. Thus, the cells would not be connected through the vertices of triangular faces but through the stems between the square faces and so the deformation would spread throughout the structure in a different manner.

In a future research, the optimized micro-structural models will be used for production of samples for experimental verification of the numerical results during a series of validation experiments.

\section{ACKNOWLEDGEMENTS}

The research has been supported by project INAFYM within Operational Programme Research, Development and Education (project no. CZ.02.1.01/0.0/0.0/16_019/0000766).

\section{REFERENCES}

[1] Y. Sugimura, J. Meyer, M. Y. He, et al. On the mechanical performance of closed cell al alloy foams. Acta Materialia 1997.

[2] P. Koudelka, O. Jiroušek, T. Fíla, T. Doktor. Compressive properties of auxetic structures produced with direct 3d printing. Materiali in tehnologije 2016.

[3] T. Doktor, P. Koudelka, T. Fíla, O. Jiroušek. Finite element based structural optimization of auxetic structures. Civil-Comp Proceedings 2015.

[4] R. S. Lakes. Foam structures with a negative poisson's ratio. Science 1987.

[5] K. K. Saxena, R. Das, E. P. Calius. Three decades of auxetics research - materials with negative poisson's ratio: A review. Advanced Engineering Materials 2016. 
[6] R. Critchley, I. Corni, J. Wharton, et al. The preparation of auxetic foams by three-dimensional printing and their characteristics. Advanced Engineering Materials 2013.

[7] J. B. Choi, R. S. Lakes. Nonlinear analysis of the Poisson's ratio of negative Poisson's ratio foams. Journal of Composite Materials 1995.

[8] X. Hou, V. V. Silberschmidt. Metamaterials with Negative Poisson's Ratio: A Review of Mechanical Properties and Deformation Mechanism. Springer, Cham, 2015.

[9] P. Koudelka, O. Jiroušek, J. Valach. Determination of mechanical properties of metal foams using microstructural models. 2011.
[10] X.-T. Wang, B. Wang, Z.-H. Wen, L. Ma.

Fabrication and mechanical properties of cfrp composite three-dimensional double-arrow-head auxetic structures. Composites Science and Technology 2018.

[11] P. Koudelka, P. Zlámal, D. Kytýř, et al. On the modelling of the compressive behaviour of metal foams: A comparison of discretization schemes. Proceedings of the Fourteenth International Conference on Civil, Structural and Environmental Engineering Computing 2013. 